\title{
Alterstice
}

Revue internationale de la recherche interculturelle

International Journal of Intercultural Research

Revista International de la Investigacion Intercultural

\section{Exercer la pédiatrie en contexte multiculturel : une approche complémentariste du rapport institutionnalisé à l'Autre, de Yvan Leanza}

\section{Caterine Bourassa-Dansereau}

Volume 3, numéro 1, 2013

URI : https://id.erudit.org/iderudit/1077504ar

DOI : https://doi.org/10.7202/1077504ar

Aller au sommaire du numéro

Éditeur(s)

Alterstice

ISSN

1923-919X (numérique)

Découvrir la revue

Citer ce compte rendu

Bourassa-Dansereau, C. (2013). Compte rendu de [Exercer la pédiatrie en contexte multiculturel : une approche complémentariste du rapport institutionnalisé à l'Autre, de Yvan Leanza]. Alterstice, 3(1), 103-108.

https://doi.org/10.7202/1077504ar d'utilisation que vous pouvez consulter en ligne. 


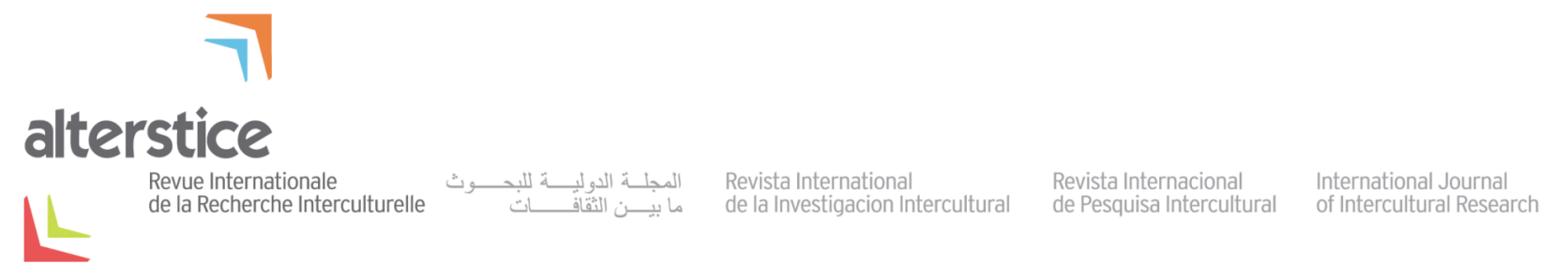

NOTE DE LECTURE

\title{
Exercer la pédiatrie en contexte multiculturel : une approche complémentariste du rapport institutionnalisé à l'Autre, de Yvan Leanza
}

\author{
Caterine Bourassa-Dansereau ${ }^{1}$
}

\author{
Rattachement de l'auteure \\ ${ }^{1}$ Université du Québec à Montréal (UQAM) et Groupe d'études et de recherche axées sur la communication \\ internationale et interculturelle (GERACII), Québec, Canada
}

\section{Correspondance}

bourassa-dansereau.caterine@uqam.ca

\section{Références de l’ouvrage :}

Leanza, Y. (2011). Exercer la pédiatrie en contexte multiculturel: une approche complémentariste du rapport institutionnalisé à l'Autre. Chêne-Bourg : Georg éditeur, 215 p.

\section{Pour citer cet article :}

Bourassa-Dansereau, C. (2013). Exercer la pédiatrie en contexte multiculturel: une approche complémentariste du rapport institutionnalisé à l'Autre [Note de lecture]. Alterstice, 3(1), 103-108.

\section{Présentation générale}

Cet ouvrage de 215 pages présente la démarche et les résultats de la recherche doctorale réalisée par Yvan Leanza à l'Hôpital de l'enfance de Lausanne (HEL), plus précisément à la policlinique de cette institution pédiatrique. Le milieu de recherche est caractérisé par la population qu'il accueille : une population défavorisée parmi laquelle les enfants de migrants sont largement représentés. Face à cette diversité, l'auteur s'est posé cette question qui oriente l'ensemble de sa démarche : « quel accueil est fait aujourd'hui à la différence (culturelle) ${ }^{1}$ dans le monde médical? » (p. 20).

\section{L'auteur}

Yvan Leanza est docteur en sciences de l'éducation (Université de Genève). II est professeur agrégé à l'École de psychologie de l'Université Laval où il codirige le laboratoire Psychologie et Cultures. Il est membre du Conseil de I'Association pour la recherche interculturelle (ARIC), du Centre interuniversitaire d'étude sur les langues, les arts et les traditions (CELAT), ainsi que des groupes de recherche Migrations et ethnicité dans les interventions en santé et

\footnotetext{
${ }^{1}$ Bien que la différence soit aussi culturelle, l'auteur mettra entre parenthèses tout au long de l'ouvrage le terme «culturel» pour signifier que la différence est «le produit d'une interaction» et pour souligner «le caractère situé et interactif de la production des différences» (p. 20).
} 
services sociaux (METISS, CSSS de la Montagne, Montréal) et Proximité et recherche sur les interventions, les services et leurs modalités (PRISM, CSSS de la Vieille-Capitale).

\section{Chapitre 1 - De la genèse et du contenu de cette recherche (p. 19-27)}

L'ouvrage de Leanza s'ouvre sur un récit qui illustre le propos de l'ouvrage et ses différentes dimensions : I'histoire d'une jeune femme immigrante et allophone qui décéda dans un établissement de santé suisse dans les années 1970. Le personnel médical n'avait alors pas pris au sérieux les douleurs ressenties par la jeune femme, malgré la présence d'une interprète improvisée (la mère de l'auteur, infirmière dans cet établissement) qui avait tenté de « faire le pont » entre la patiente et le personnel médical.

À partir de ce récit, l'auteur précise les questionnements qui orientent sa démarche de recherche:

- Quelle est la place de la différence (culturelle) dans le monde médical et comment est-elle accueillie?

- Comment reconnaît-on et accueille-t-on les différents types de savoirs (profanes et savants) dans le milieu médical?

- Comment est entendue et reçue la voix des patients qui ne peuvent s'exprimer dans celle des soignants?

- Quels sont la place et le rôle des interprètes-médiatrices culturelles ${ }^{2}$ dans le monde médical?

\section{Cadre théorique et posture}

Leanza mobilise au cours de sa démarche différentes disciplines qu'il place sous l'égide des approches interculturelles. Du fait de leur nature complémentariste, les approches interculturelles permettent «le dépassement des limites explicatives et méthodologiques propres à une discipline » (p. 22) et ont la particularité et l'avantage « de faire la part belle à la complémentarité entre disciplines ayant pour objet de réflexion les groupes humains [...] et celles qui centrent leur réflexion sur les interactions interindividuelles, voire sur les individus seuls » (p. 22).

La posture complémentariste permet à l'auteur de construire du sens différemment :

Cette posture me permet, tout en restant attentif à la dimension symbolique, de passer du collectif (comment l'institution, la biomédecine et la société donnent-elles sens à la différence?) à l'individuel (comment individuellement chacun des pédiatres et interprètes donne-t-il sens à sa pratique et à la différence?), puis de les articuler. (p. 23)

L'ouvrage s'organise autour du cadre théorique de la "niche d'activités professionnelles » (p. 53) développé par l'auteur. C'est à partir de ce point d'ancrage que Leanza explore différentes dimensions de la pratique pédiatrique en contexte interculturel et analyse les résultats obtenus.

\section{Chapitre 2 - Pédiatrie contemporaine, psychologie interculturelle et démarche de recherche (p. 27-57)}

Leanza situe dans un premier temps sa recherche dans le contexte suisse : celui d'un pays d'immigration et de diversité ethnoculturelle. Plus précisément, il place sa démarche dans le cadre pédiatrique contemporain où la rencontre entre médecins, parents et enfants " n'est pas le lieu de seuls enjeux thérapeutiques. Ce sont des rapports sociaux plus larges qui [y] sont mis en scène et interprétés » (p. 31-32). L'auteur précise en effet que les pédiatres sont en quelque sorte tributaires sur les plans social et individuel du maintien des normes considérées comme "naturelles" concernant la santé des enfants. L'étude des examens de prévention effectués par les médecins auprès des enfants migrants (terrain d'étude de Leanza) permet donc à l'auteur d'accéder à un double enjeu : la mise en scène des ethnothéories pédiatriques face aux enfants et à leurs parents, ainsi que l'accueil qui est réservé à ces derniers en regard de leurs différences (culturelles). Leanza peut dès lors observer « l'expérience de la rencontre avec des familles migrantes telle qu'elle est vécue par des pédiatres dans leurs pratiques de prévention » (p. 28).

\footnotetext{
${ }^{2}$ Les interprètes-médiatrices culturelles sont des traductrices de la langue, mais aussi des médiatrices (inter)culturelles qui permettent le dialogue entre patients et médecins (p. 20).
} 


\section{Démarche de recherche}

Pour Leanza, la culture est envisagée comme une construction permettant de " faire sens » et à travers laquelle l'individu navigue et fait des choix : « il y a ainsi un rapport vivant qui s'établit entre les individus et ces matrices de sens dans lesquelles ils évoluent » (p. 40).

L'auteur souligne de plus les rapports de force et les relations de pouvoir qui caractérisent les interactions humaines, particulièrement dans le cadre institutionnalisé des soins médicaux qui orientent les rapports entre soignants et soignés. En ce sens, la démarche de Leanza vise à

effectuer une analyse de ces interactions particulières que sont les examens de suivi développemental avec interprètes qui tiennent compte des contextes institutionnel (la policlinique), professionnel (la biomédecine) et social (Lausanne et plus largement la Suisse) dans lesquels ces interactions prennent place. Cependant, c'est aussi l'interprétation des acteurs euxmêmes que je me propose de mettre en évidence, en d'autres termes, leurs choix d'action et la signification qu'ils leur donnent. (p. 41)

Comme principal outil, l'auteur développe conséquemment la théorie de la niche d'activités professionnelles: "Cette façon de conceptualiser l'activité du professionnel permet de dépasser les limites propres aux théories de la psychologie sociale (interculturelle) qui négligent à la fois les aspects contextuels de l'interaction et les aspects subjectifs » (p. 54). Grâce à celle-ci, Leanza vise à accéder au sens donné par le médecin à la différence lors des interactions avec les enfants et leurs parents, au sens donné aux ethnothéories dont il est porteur, au sens donné à sa propre pratique et au sens donné au contexte dans lequel il travaille.

\section{Chapitre 3 - Le contexte : I'institutionnalisation et « l'accueil » de la différence (p. 57-71)}

Dans ce contexte de grande diversité qui caractérise la policlinique, Leanza souligne que le fonctionnement quotidien n'est pourtant pas toujours favorable à l'accueil de la différence. II mentionne "comment certaines caractéristiques du fonctionnement quotidien marquent le rapport à l'Autre » (p. 59) et indique à ce propos quatre particularités institutionnelles (p.60-67): les initiatives favorisant l'accueil interculturel, les articulations difficiles entre les différents professionnels, les contraintes de temporalité qui exercent une pression et mènent parfois à la rupture du lien soignant-soigné, ainsi que le fait que la policlinique soit aussi un lieu de formation pour les futurs médecins.

Suite à ses observations, l'auteur met en lumière un paradoxe au sein de l'institution : il y a à la policlinique une volonté de changement et un désir d'accueillir et de reconnaître la différence, et pourtant " [...] les analyses montrent que ce souhait d'innover rencontre une résistance forte [...]. II s'agit de la force d'inertie propre à tout système » (p. 70). Pour Leanza, il s'agit de la force d'inertie inhérente à l'hôpital lui-même, à laquelle s'ajoute celle associée à la culture biomédicale.

\section{Chapitre 4 - Les consultations de prévention : la pratique et les ethnothéories (p. 71-121)}

Leanza présente dans ce chapitre les résultats des observations réalisées lors de consultations de prévention effectuées par les médecins. Son corpus est composé de 21 consultations ayant eu lieu en présence d'interprètesmédiatrices culturelles et des 36 incidents critiques qui en ont émergé. Trois processus principaux y sont observés (p. 71) : comment la différence se fait voir et entendre dans le déroulement d'une consultation, en quoi l'interprète-médiatrice culturelle est un support pour faire émerger cette différence et comment la différence est traitée dans la communication entre médecins et patients. Nous les expliquons ici plus en détail.

L'auteur aborde dans un premier temps la place de la différence lors des consultations et souligne que, quelle que soit l'origine des parents, les consultations de prévention sont immuables. La pratique est donc homogène malgré la diversité des patients de la policlinique. Pour l'auteur, ceci démontre la valeur symbolique associée à la répétition du schéma de consultation qui participe à ce que l'enfant rencontré soit un peu moins "autre " et qu'il devienne " des nôtres » : " II y a [...] volonté, plus ou moins consciente, de l'institution et de ses agents de modeler la fabrication des petits d'hommes » (p. 81). 
Concernant le rôle des interprètes-médiatrices culturelles, Leanza examine si ces dernières ne sont que responsables de la traduction des mots ou si elles participent aussi à la mise en place d'un "pont " entre les cultures. L'auteur rappelle que « [...] leur fonction les autorise à intervenir aussi bien au niveau de la langue que du sens que peuvent revêtir certaines pratiques parentales ou biomédicales. Elles peuvent être un lien vivant entre les cadres de référence » (p. 82). L'auteur constate par ailleurs qu'il n'y a pas beaucoup de place accordée au « monde du vécu » lors des rencontres de consultation et que le travail des interprètes relève moins de la « médiation » que de la «traduction ».

En abordant l'émergence de la différence dans la communication entre médecins et patients, Leanza constate que l'entretien médical est souvent un lieu de conflit entre deux façons de construire du sens: le langage associé au vécu (langage commun, faits médicaux contextualisés) et celui de la biomédecine (langage spécialisé, exclusion des faits contextualisés) (p.96). Il mentionne que "les schémas privilégiant la voix de la biomédecine dominent» (p. 99) lors des consultations et que les interprètes-médiatrices culturelles interrompent parfois elles-mêmes la communication "du vécu » engagée par les parents et donnent souvent un poids plus important au langage normatif et biomédical (p. 113-114).

En conclusion, Leanza constate que :

À ce point de l'analyse, je considère que les consultations étudiées, de par leur caractère séquentiel rigide, peu centré sur les pratiques des parents (sur le monde vécu) et par leur "neutralisation " presque généralisée des rôles des interprètes associées au monde vécu, ainsi que par le caractère systématique de répétition de la norme biomédicale sur ces thèmes éducatifs à haute teneur symbolique, fonctionnent comme un rite d’assimilation (p. 119).

\section{Chapitre 5 - l'expérience vécue des médecins (p. 121-143)}

En concordance avec le cadre théorique de la niche d'activités professionnelles, l'auteur réalise des « entretiens d'autoconfrontation » avec les médecins ayant participé aux consultations filmées. Leanza souhaite ainsi accéder au sens que ces derniers donnent à leurs propres pratiques, et ce, à quatre niveaux (p. 124) :

- «Comment les médecins comprennent-ils les pratiques parentales (sens de la différence)? »

- «À quel point adhèrent-ils aux normes qu'ils ont à transmettre et aux modèles de pratiques prescrits par I'institution et la biomédecine (rapport aux ethnothéories)? "

- «Quel style de pratique estiment-ils mettre en action (rapport à la pratique)? »

- «Comment vivent-ils leur condition de travail (rapport au contexte)?»

En s'inscrivant dans une approche de théorisation ancrée, l'auteur fait émerger de ces entretiens des typologies correspondant aux différentes formes que peut prendre le rapport à l'Autre dans le cadre de la pratique médicale. Ces typologies non exclusives se situent dans des continuums sur lesquels les médecins sont mobiles.

Leanza constate à ce propos que le rapport institutionnel à l'Autre qu'entretiennent les médecins oscille entre des processus de "normalisation " (forte conviction face aux modèles médicaux, imposition de normes, acceptation des conditions de travail, etc.) et de "particularisation » (doutes sur les ethnothéories, négociation, malaise concernant les conditions de travail, etc.). Il mentionne toutefois que la normalisation reste dominante. L'auteur souligne de plus la présence de «tensions de normalisation » qui « semblent être la résultante d'un conflit entre la volonté de perpétuer un modèle imposé par l'institution, et l'expérience que ce modèle n'est pas adéquat pour la situation rencontrée au quotidien » (p.141). Pour l'auteur, ces états semblent induire des changements chez les médecins et dans leur pratique et « [...] laissent penser que le rapport à l'Autre (culturel) en contexte professionnel suit une évolution. Le point de départ serait la normalisation forte et l'aboutissement la particularisation forte " (p. 141). 


\section{Chapitre 6 - l'expérience vécue par les interprètes-médiatrices culturelles (p. 143-161)}

Leanza rend compte des résultats obtenus lors d'entretiens d'autoconfrontation réalisés auprès des interprètesmédiatrices culturelles, à la demande explicite de ces dernières. Ces résultats permettent d'accéder au sens qu'elles donnent à leur propre pratique.

Les interprètes-médiatrices culturelles rencontrées par Leanza dénoncent dans un premier temps le manque de ressources et le peu de reconnaissance accordée à leur travail (p. 145-148). L'auteur précise qu'elles perçoivent leur rôle comme un vecteur d' " intégration ", de "soutien " et " d'accompagnement ". Elles-mêmes se perçoivent comme étant des "informatrices culturelles " plutôt que des " médiatrices culturelles ». Elles s'associent même parfois à des " agentes du système médical ", car elles notent que les transferts d'informations et de sens vont du médecin vers le patient, et ne se font pas en direction inverse.

En regard de ces résultats, Leanza souligne la nécessité de soutenir et de valoriser le duo interprète-médecin dans l'institution. Dans l'état actuel des choses, les interprètes-médiatrices culturelles participent en effet surtout à l'établissement et au transfert des normes biomédicales et ne favorisent pas le dialogue culturel entre médecins et patients, et ce, malgré le rôle de « passeur culturel » qui devrait caractériser leur travail.

\section{Chapitre 7 - Des soins en tension entre universalisme et particularisme (p. 161-185)}

Dans ce dernier chapitre, Leanza fait le point sur les résultats obtenus et propose des pistes de réflexion à leur sujet.

Comme premier constat, Leanza affirme que la culture biomédicale oscille entre «objectivation » (pensée rationaliste qui exclut les caractéristiques familiales, socioculturelles et émotionnelles) et "subjectivation » (ouverture vers le cadre de référence du patient) (p. 163). L'auteur souligne qu'il y a à la policlinique une volonté d'aller vers une médecine plus subjective, mais qu'une inertie institutionnelle se dresse et fait obstacle, " [...] cette volonté d'intégrer se heurte à des fonctionnements "habituels", propres à toute institution hospitalière, qui l'entravent, voire la brisent » (p. 165).

L'auteur présente de plus certains constats sur la pratique des médecins. Chez ces derniers, le " bon savoir » est le savoir de la biomédecine et non celui du vécu. Il rappelle que la majorité des médecins considère que les pratiques parentales "différentes » sont le résultat de carences. En ce sens, la pratique médicale a donc surtout un rôle de régulation et de normalisation.

De façon générale, l'auteur statue en effet qu'une vision assimilationniste est toujours présente dans le monde médical et qu'elle s'exprime lors des entretiens analysés : "La "mission" implicite de ces consultations, en l'état où je les ai observées, est de faire en sorte que les parents "fabriquent" des enfants dans la norme, des enfants comme les autres et non différents » (p. 169).

Toutefois, l'auteur entrevoit la venue de changements dans la pratique, entre autres en réaction à certains malaises vécus par les médecins :

[...] les malaises sont une nécessité à la construction d'un nouveau sens et/ou de nouvelles pratiques, plus précisément une pratique centrée sur les parents et les enfants. [...] ces états psychologiques peuvent servir de déclencheurs à un processus de changement. (p. 167)

Leanza souligne qu'il faudra toutefois dépasser le "malaise individuel » et favoriser de nouvelles pratiques organisationnelles pour permettre l'accueil interculturel et de véritables changements systémiques. L'auteur conclut ce chapitre avec différentes pistes d'action pour aller dans ce sens et grâce auxquelles « il s'agit de prendre au sérieux la culture, les univers de sens et les expériences des professionnels oeuvrant en contextes multiculturels » (p. 181). 


\section{Commentaires}

La démarche de Yvan Leanza permet d'accéder aux rouages de l'accueil interculturel dans une grande institution médicale et de comprendre comment et pourquoi certains écueils peuvent apparaître et subsister. En ce sens, l'ouvrage permet de mettre en lumière les écarts entre les discours sur l'interculturel (ce que les professionnels de l'institution souhaitent) et les pratiques (les façons dont se déroulent effectivement les rencontres entre patients, médecins et interprètes). À ce propos, l'accès aux perceptions des médecins et au sens qu'ils donnent à leur propre pratique est essentiel afin de mieux comprendre les processus régissant les relations interculturelles entre médecins et patients. L'approche subjectiviste de la démarche de Leanza est ainsi un atout majeur de l'ouvrage.

Par ailleurs, l'ouvrage est très bien écrit et le lecteur accompagne avec plaisir et intérêt Leanza à travers sa démarche et sa réflexion.

Nous soulevons toutefois deux points de réflexion en conclusion.

Dans un premier temps, nous rappelons la définition nuancée que donne l'auteur de la culture. Ce dernier la situe entre autres à l'intérieur de rapports de force et de relations de pouvoir plus larges qui encadrent et orientent à la fois la " culture " et les rencontres interculturelles qui s'ensuivent. Cette dimension de la culture est pourtant peu abordée par l'auteur lorsqu'il observe et analyse les relations entre médecins et patients. À ce propos, il nous semble que la vision des médecins concernant par exemple les " carences parentales " associées à la différence prend en effet racine dans des rapports sociaux plus larges, non seulement liés au contexte institutionnel et médical, mais aussi au contexte national et international (par exemple, aux rapports entre groupes majoritaire et minoritaires).

Dans un second temps, nous soulignons l'intérêt pour une prochaine recherche de s'intéresser aussi au point de vue des patients : ces derniers restent en effet sans voix dans l'ouvrage de Leanza. Si l'objectif de la démarche de l'auteur était de se centrer sur l'activité et l'expérience des médecins, il nous semble que l'accès à la vision des patients permettrait d'affiner et d'enrichir les analyses sur le rapport à la différence et son accueil en milieu médical. 